\title{
Periodicidade de oviposição de fêmeas de Aedes aegypti (Linnaeus, 1762) (Diptera: Culicidae) em laboratório e campo
}

\author{
Periodicity of oviposition of females of Aedes aegypti (Linnaeus, 1762) \\ (Diptera: Culicidae) in laboratory and field
}

\author{
Adriana dos Santos Gomes ${ }^{1}$, Célia J. de Sá Sciavicoe élvaro Eduardo Eiras ${ }^{1}$
}

\begin{abstract}
RESUMO
o objetivo deste trabalho foi determinar o padrão gonotrófico de fêmeas de Aedes aegypti em condições de laboratório e de campo. Fêmeas copuladas e alimentadas com sangue de galinha foram transferidas para gaiolas de polipropileno e oferecidos diferentes substratos de oviposição (papel sulfite, filtro, manteiga e toalha). O papel filtro recebeu significativamente maior $(40,4 \%)$ deposição de ovos do que os demais substratos. Observou-se que maior (35,7\% dia modal) oviposição ocorreu ao terceiro dia, após alimentação sangüínea. Foi observada a oviposição das fêmeas a cada duas horas de intervalo, durante o fotoperíodo em laboratório e em campo por meio de ovitrampas. Os resultados de laboratório demonstraram que maior deposição de ovos ocorreu entre a $9^{a}-12^{a}$ hora da fotofase e $1^{a}-2^{a}$ hora de escotofase. Em campo maior número de ovos ocorreu entre $9^{a}-12^{a}$ hora de fotofase e na $1^{a}$ - $4^{a}$ hora de escotofase. Estes resultados indicam que Aedes aegypti exibiu um padrão na periodicidade de oviposição e podem subsidiar em programas de monitoramento e ou controle do inseto vetor. Sugere-se que, em campo, por exemplo, armadilhas de oviposição (ovitrampa) devem ser instaladas no período da manhã pois as capturas ocorrem a partir do período da tarde.
\end{abstract}

Palavras-chaves: Oviposição. Aedes aegypti. Fotoperíodo. Substratos.

\begin{abstract}
The object of this work was to determine of gonotrophic diel pattern of female Aedes aegypti in laboratory and field conditions. Three day-old female mosquitoes were the fed on chicken blood and transferred to bioassay cages. Four oviposition substrates were offered: paper sulfite, filter, butter and towel. The results showed that filter paper received a significantly higher (40.4\%) percentage of deposited eggs than the other oviposition substrates. After their first blood meal, females started to oviposit on the $3^{\text {rd }}$ model day; $35.7 \%$ of the total number of eggs deposited. The oviposition diel patterns of females were observed every two hours during the photoperiod in the laboratory and in the field. In the laboratory, the periodicity of oviposition showed that the highest egg deposition occurred during the $9^{\text {th }}-12^{\text {th }} \mathrm{h}$ of photophase and $1^{\text {st }}-2^{\text {nd }} \mathrm{h}$ of scotophase. In the field, the highest egg deposition occurred during the $9^{\text {th }}-12^{\text {th }} \mathrm{h}$ of photophase and $1^{\text {st }}-4^{\text {th }} \mathrm{h}$ of scotophase. These results point out that Aedes aegypti showed an oviposition periodicity pattern that can subsidize monitoring and or control of vector insect. Itis suggested that ovitraps should be placed in the field during the morning hours since the captures occur during afternoon.
\end{abstract}

Key-words: Oviposition. Aedes aegypti. Photoperiod. Substrates.

\footnotetext{
1. Departamento de Parasitologia do Instituto de Ciências Biológicas da Universidade Federal de Minas Gerais, Belo Horizonte, MG. Apoio financeiro: IFS, CAPES, CNPq e FUNASA.

Endereço para correspondência: Dr. Álvaro Eduardo Eiras. Dept ${ }^{\circ}$ Parasitologia/ICB/UFMG. Av. Presidente Antônio Carlos 6627, Caixa Postal 486, Pampulha, 31270-901 Belo Horizonte, MG

Tel: 5531 3499-2976

e-mail: alvaro@icb.ufmg.br

Recebido para publicação em 14/7/2006

Aceito em 29/5/2006
} 
O padrão de oviposição de Aedes aegyptivem sendo estudado por vários autores 813141525 . Os diversos comportamentos realizados por fêmeas nos criadouros podem estar determinados por fatores exógenos especialmente a chuva, a umidade relativa do ar, a temperatura e a velocidade do vento ${ }^{4}$. A localização e a seleção de um possível criadouro para a oviposição envolvem respostas visuais, olfativas e táteis. Fêmeas de A. aegypyti utilizam de atraentes de oviposição presentes em infusões de gramíneas ${ }^{6022}$ e contendo larvas co-específicas ${ }^{2}{ }^{18} 28$. Dentre os fatores físicos, comprimento de onda, intensidade de luz refletida na água ou substrato ${ }^{17}$ absorção de água e aspereza do substrato ${ }^{12}$, podem determinar a escolha de criadouros para a deposição de ovos.

Fêmeas de $A$. aegypti tendem a realizar a oviposição no interior de recipientes que armazenam água de torneira ou de chuvas $^{16}$, embora já tenha sido relatada a capacidade em realizarem oviposição na superfície da água ${ }^{15}{ }^{1924}$. Em laboratório foram observadas a influência da coloração do substrato e do fotoperíodo na oviposição desses mosquitos; substratos mais escuros vêm sendo apontados como mais atrativos para algumas espécies, e os picos de oviposição parecem estar relacionados aos períodos de fotofase $\mathrm{e}^{379126}$. Estudos relacionados ao padrão de oviposição de fêmeas de $A$. aegypti são relevantes para a compreensão de aspectos biológicos e ecológicos desse vetor, pois proporcionam dados que podem subsidiar os métodos atuais de monitoramento e controle da espécie. 0 objetivo deste trabalho foi determinar a periodicidade de oviposição para fêmeas de A. aegypti em condições de laboratório e campo.

\section{MATERIAL E MÉTODOS}

Criação e manutenção dos insetos. A sala de criação foi mantida com umidade relativa de $70 \pm 5 \%$ e fotoperíodo 12L:12E ${ }^{22}$. A colônia de A. aegypti foi obtida através de ovos coletados no Campus Pampulha da UFMG, utilizando armadilhas de oviposição. Ovos de A. aegypti foram colocados em recipientes plásticos de $500 \mathrm{ml}$ com água desclorada, para eclosão das larvas. As larvas de $1^{\circ}$ estádio foram transferidas para recipientes plásticos $(28 \times 10 \times 7 \mathrm{~cm})$ com aproximadamente $700 \mathrm{ml}$ de água desclorada e receberam, como alimento, dieta em pó (ração de camundongo, triturada, autoclavada e peneirada) até atingirem o estádio de pupa. As pupas foram diariamente transferidas para vasilhames plásticos $(10 \times 6 \mathrm{~cm})$ com água desclorada e colocadas em gaiolas de acrílico $(40$ x 40 x $40 \mathrm{~cm})$, até emergência dos adultos, permanecendo os machos junto com as fêmeas para a cópula. Os insetos adultos foram alimentados com solução de sacarose $10 \%$, até 24 horas antes do repasto sanguíneo. Fêmeas com três dias de idade, após a emergência, receberam repasto sangüíneo (sangue de galinha Gallus gallus, contendo heparina sódica) por meio de alimentador artificial $^{1}$, entre o período da $1^{\mathrm{a}} \mathrm{e} 2^{\mathrm{a}}$ hora de fotofase.

Seleção do substrato de oviposição para fêmeas de Aedes aegypti. Foram avaliadas 180 fêmeas (10 fêmeas/gaiola), durante nove dias consecutivos, em gaiolas de polipropileno (30 x 30 x 30cm) (Bugdorm-I, Mega View Science Education
Services, Taiwan), providas com solução sacarose 10\%. Quatro tipos de papéis brancos (filtro, manteiga, sulfite e toalha) foram testados como substratos de oviposição, revestindo internamente recipientes plásticos transparentes $(7,5 \times 4,5 \times 5 \mathrm{~cm})$ contendo água. Estes foram substituídos diariamente na $1^{\mathrm{a}}$ hora de fotofase sendo que, as posições destes foram alternadas a cada repetição. Em todos os bioensaios de laboratório, avaliou-se a presença de ovos no substrato de oviposição e na superfície da água de cada recipiente. Os ovos foram contados à lupa estereoscópica. Foram realizadas nove repetições.

Padrão diário de oviposição de fêmeas de Aedes aegypti em laboratório. A partir do primeiro dia após repasto sangüíneo, 100 fêmeas foram selecionadas e distribuídas em 10 insetos/gaiola de polipropileno semelhante ao experimento anterior. Cada gaiola recebeu 0 substrato que obteve melhor oviposição no teste anterior. A oviposição foi observada durante 10 dias consecutivos e os ovos depositados no substrato foram quantificados diariamente utilizando a metodologia anteriormente citada. Foram realizadas 10 repetições.

Periodicidade de oviposição em laboratório. Foram avaliadas 100 fêmeas de $A$. aegypti durante $03^{\circ}$ e $4^{\circ}$ dia após repasto sangüíneo, sendo formado 10 grupos de insetos/gaiola de polipropileno. 0 substrato de oviposição foi oferecido na $1^{\text {a }}$ hora de fotofase e substituído a cada duas horas do fotoperíodo. O número de ovos foi determinado seguindo metodologia dos testes anteriores. Foram realizadas 10 repetições.

Oviposição de Aedes aegypti em campo (ambiente peridomiciliar). Os bioensaios foram realizados nas dependências do Instituto de Ciências Biológicas (ICB), Universidade Federal de Minas Gerais (UFMG), Campus Pampulha, Belo Horizonte (MG), durante o mês de março de 2002. Utilizou-se 20 armadilhas de oviposição (ovitrampa) que consistiram de recipiente plástico, pretofosco, com capacidade de $700 \mathrm{ml}$. Cada armadilha recebeu aproximadamente $350 \mathrm{ml}$ de infusão diluída $10 \%$ da gramínea Panicum maximum ${ }^{202}$, e uma palheta de madeira $(3 \mathrm{x} 13 \mathrm{x}$ $0,5 \mathrm{~cm})$, presa verticalmente por clips, como substrato de oviposição. As armadilhas foram distribuídas e instaladas no solo, com uma distância mínima entre estas de dois metros, de forma casualizada na $1^{\text {a }}$ hora de fotofase $(05: 15 \mathrm{~h})$, em área de pouca vegetação, no peridomicílio durante 0 fotoperíodo: fotofase (nascer do sol) e escotofase (ocaso do sol) por quatro dias consecutivos. Estes horários foram fornecidos pelo Laboratório de Astrofísica, Departamento de Física, Instituto de Ciências Exatas (ICEX) da Universidade Federal de Minas Gerais, Campus da Pampulha, Belo Horizonte (MG). As palhetas foram substituídas a cada duas horas durante o fotoperíodo e aquelas contendo ovos foram colocadas em recipientes $(10 \times 6 \mathrm{~cm})$ com água para eclosão das larvas; para posterior identificação ${ }^{10}{ }^{26}$. Não foi avaliada neste experimento a presença de ovos na superfície da infusão devido observações anteriores que demonstram a ausência de ovos na infusão. Foram realizadas 20 repetições a cada horário do fotoperíodo, totalizando 960 palhetas recolhidas. 
Análise Estatística. Os números de ovos depositados em todos os substratos e na superfície da água dos recipientes de oviposição foram submetidos ao teste de normalidade (Kolmogorov-Smirnov). Como os dados não obedeceram a uma distribuição normal, foram então, submetidos ao teste de Kruskal Wallis $(\mathrm{p}<0,05)$.

\section{RESULTADOS}

Escolha do substrato de oviposição. Em laboratório a atividade de oviposição ocorreu em todos os substratos oferecidos (Tabela 1). Os resultados demonstraram que 0 papel filtro foi o substrato que significativamente $(\mathrm{p}<0,05)$ recebeu o maior número de ovos depositados (5.982 ovos). Os papeis sulfite, manteiga e toalha receberam 2.422, 2.937 e 3.418 ovos, respectivamente. As fêmeas de A. aegypti demonstraram maior atividade de oviposição durante 0 período de escotofase (11.092 ovos) do que fotofase (3.667 ovos). Durante a fotofase, o papel filtro $(1.214$ ovos $)$ e 0 papel toalha (1.200 ovos), receberam significativamente $(\mathrm{p}<0,05)$ maior oviposição, enquanto na escotofase 0 papel filtro também recebeu significativamente o maior (4.768) número de ovos depositados (Figura 1).

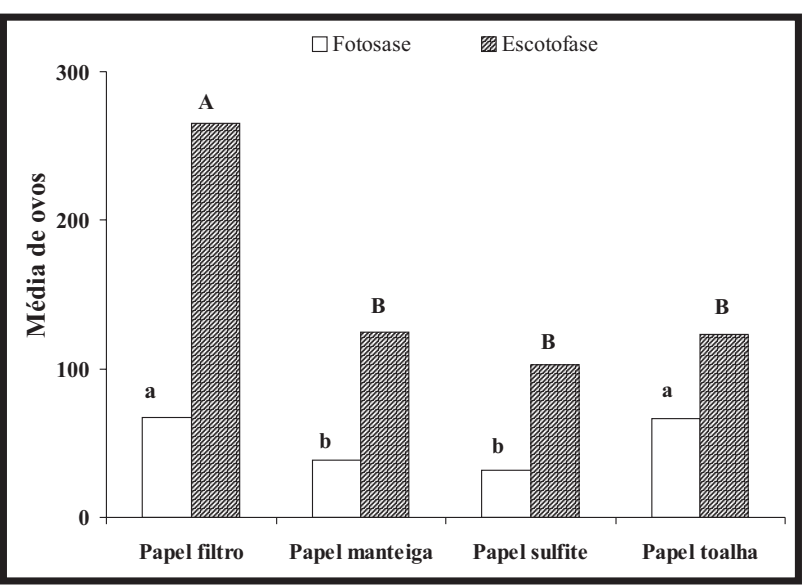

Figura 1 - Média de ovos de fêmeas grávidas do mosquito Aedes aegypti depositados durante fotoperíodo em diferentes substratos de oviposição. ( $N=18$ repetições, Kruskal Wallis, $p<0,05$ )

Letras maiúsculas indicam diferença significativa entre si para o período da escotofase, enquanto minúsculas indicam diferença significativa para o período de fotofase.
As fêmeas de Aedes aegypti realizaram oviposições tanto no substrato quanto na superfície da água dos recipientes de oviposição durante o fotoperíodo (Tabela 1). Durante a fotofase, 0 recipiente revestido internamente com papel toalha (33\%) recebeu o maior percentual de ovos depositados na parede, enquanto o maior $(31,9 \%)$ percentual de ovos foi observado na superfície da água no recipiente contendo papel manteiga.

Na escotofase, o papel filtro e o papel toalha receberam maior percentual na deposição de ovos $65,9 \%$ e 53,3\%, respectivamente, enquanto os recipientes com papel manteiga $(44,2 \%)$ e papel sulfite $(65 \%)$ receberam maior oviposição na superfície da água.

Determinação do padrão temporal diário de oviposição de fêmeas de Aedes aegypti em laboratório. As fêmeas de $A$. aegypti não exibiram nenhuma atividade de oviposição nas 48 horas subseqüentes à alimentação sangüínea (Figura 2). Verificou-se que, no terceiro dia após 0 repasto sangüíneo, ocorreu significativamente $(\mathrm{p}<0,05)$, o maior percentual de ovos depositados $(35,7 \%)$, em relação aos dias subseqüentes. A oviposição no quarto, no quinto e no sexto dia demonstrou-se estável não havendo diferença estatística entre estes $(p>0,05)$. Um decréscimo na oviposição ocorreu a partir do sétimo dia, entretanto, a oviposição aumentou no oitavo dia e gradativamente diminuiu entre o nono e o décimo dia de oviposição (Figura 2).

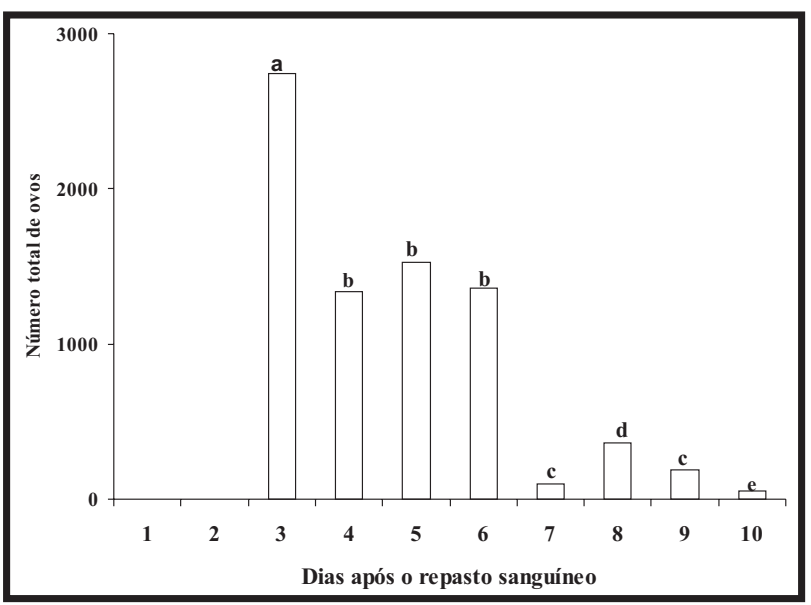

Figura 2 - Número total de ovos depositados por fêmeas grávidas do mosquito Aedes aegypti em laboratório, após primeiro repasto sangüíneo ( $N=10$ repetições, Kruskal Wallis $p<0,05$ ).

Histogramas seguidos de letras diferentes entre si indicam diferença significativa .

Tabela 1 - Número total e percentagem de ovos de fêmeas grávidas do mosquito Aedes aegypti, depositados na parede dos diferentes substratos de oviposição e na superfície da água em condições de laboratório.

\begin{tabular}{|c|c|c|c|c|c|c|c|c|c|}
\hline \multirow[b]{3}{*}{ Substrato } & \multicolumn{4}{|c|}{ Fotofase (\%) } & \multicolumn{4}{|c|}{ Escotofase (\%) } & \multirow{3}{*}{$\begin{array}{r}\text { Total } \\
(\%)\end{array}$} \\
\hline & \multicolumn{2}{|c|}{ parede } & \multicolumn{2}{|c|}{ água } & \multicolumn{2}{|c|}{ parede } & \multicolumn{2}{|c|}{ água } & \\
\hline & $\mathrm{n}^{0}$ & $\%$ & $\mathrm{n}^{0}$ & $\%$ & $\mathrm{n}^{0}$ & $\%$ & $\mathrm{n}^{0}$ & $\%$ & \\
\hline \multirow[t]{2}{*}{ Papel filtro } & 1.060 & 17,7 & 154 & 2,6 & 3.943 & 65,9 & 825 & 13,8 & $5.982 \mathrm{a}$ \\
\hline & & & & & & & & & 100,0 \\
\hline \multirow[t]{2}{*}{ Papel toalha } & 1.130 & 33,0 & 70 & 2,0 & 1.819 & 53,3 & 399 & 11,7 & $3.418 \mathrm{~b}$ \\
\hline & & & & & & & & & 100,0 \\
\hline \multirow[t]{2}{*}{ Papel manteiga } & 362 & 12,3 & 325 & 31,9 & 950 & 32,5 & 1.300 & 44,2 & $2.937 \mathrm{~b}$ \\
\hline & & & & & & & & & 100,0 \\
\hline \multirow[t]{2}{*}{ Papel sulfite } & 75 & 3,0 & 491 & 20,3 & 284 & 11,7 & 1.572 & 65,0 & $2.422 \mathrm{~b}$ \\
\hline & & & & & & & & & 100,0 \\
\hline
\end{tabular}

Valores seguidos de letras diferentes entre si indicam diferenças significativas (Kruskal Wallis; $\mathrm{p}<0,05 ; \mathrm{N}=18$ repetições). 
Tabela 2 - Deposição de ovos por fêmeas de Aedes aegypti no papel filtro e na superfície da água, durante fotofase e escotofase ( $N=10$ repetições para cada fotoperíodo) em condições de laboratório.

\begin{tabular}{|c|c|c|c|c|c|c|c|c|c|c|}
\hline \multirow[b]{3}{*}{ Dias } & \multicolumn{4}{|c|}{$\begin{array}{c}\text { Fotofase } \\
\text { Total de ovos (\%) }\end{array}$} & \multicolumn{4}{|c|}{$\begin{array}{c}\text { Escotofase } \\
\text { Total de ovos (\%) }\end{array}$} & & \\
\hline & \multicolumn{2}{|c|}{ Substrato } & \multicolumn{2}{|c|}{ superfície água } & \multicolumn{2}{|c|}{ Substrato } & \multicolumn{2}{|c|}{ superfície água } & \multicolumn{2}{|c|}{ Total (\%) } \\
\hline & $\overline{\text { total }}$ & $(\%)$ & total & $(\%)$ & $\overline{\text { total }}$ & $(\%)$ & total & $(\%)$ & $\overline{\mathrm{n}^{0}}$ & $\%$ \\
\hline $1^{0}$ & 0 & 0,0 & 0 & 0,0 & 0 & 0,0 & 0 & 0,0 & 0 & 0,0 \\
\hline $2^{0}$ & 0 & 0,0 & 0 & 0,0 & 0 & 0,0 & 0 & 0,0 & 0 & 0,0 \\
\hline $3^{\circ}$ & 102 & 1,3 & 6 & 0,1 & 1.831 & 23,8 & 807 & 10,5 & 2.746 & 35,7 \\
\hline $4^{0}$ & 119 & 1,5 & 47 & 0,6 & 797 & 10,4 & 378 & 4,9 & 1.341 & 17,4 \\
\hline $5^{\circ}$ & 110 & 1,4 & 50 & 0,7 & 1.148 & 15,0 & 222 & 2,9 & 1.530 & 20,0 \\
\hline $6^{0}$ & 11 & 0,1 & 0 & 0,0 & 1.026 & 13,5 & 323 & 4,2 & 1.362 & 17,8 \\
\hline $7^{\circ}$ & 0 & 0,0 & 0 & 0,0 & 0 & 0,0 & 95 & 1,2 & 96 & 1,2 \\
\hline $8^{\circ}$ & 0 & 0,0 & 0 & 0,0 & 279 & 3,6 & 84 & 1,1 & 363 & 4,7 \\
\hline $9^{\circ}$ & 0 & 0,0 & 0 & 0,0 & 175 & 2,3 & 16 & 0,2 & 191 & 2,5 \\
\hline $10^{\circ}$ & 0 & 0,0 & 0 & 0,0 & 47 & 0,6 & 9 & 0,1 & 56 & 0,7 \\
\hline Total & 342 & 4,3 & 106 & 1,4 & 5.303 & 69,2 & 1.934 & 25,1 & 7.685 & 100,0 \\
\hline
\end{tabular}

Durante a fotofase, foram depositados 448 ovos nos recipientes de oviposição. Não houve diferença para o percentual de ovos depositados entre o terceiro, quarto e quinto dia após oviposição: $1,3 \%, 1,5 \%$ e $1,4 \%$, respectivamente (Tabela 2 ). No sexto dia, 0 percentual de oviposição foi extremamente reduzido $(0,1 \%)$, não ocorrendo oviposição nos dias subseqüentes de observação neste fotoperíodo (Figura 3).

Durante a escotofase, foram depositados 7.237 ovos e a maior oviposição foi observada no terceiro dia. 0 quarto, quinto e sexto dia não apresentaram diferença estatística significativa $(p>0,05)$ entre si. A oviposição observada entre o sétimo, oitavo, nono e décimo dia apresentaram um número menor de ovos, porém, indicam diferença significativa $(\mathrm{p}<0,05)$ para o oitavo dia (Figura 3).

A atividade de oviposição das fêmeas de Aedes aegypti foi observada quanto ao local de deposição de ovos no criadouro. Verificou-se que durante a fotofase as fêmeas depositaram maior quantidade de ovos no substrato de oviposição (342 ovos) do que na superfície da água (106 ovos). 0 quarto dia apresentou maior oviposição no substrato (119 ovos); a maior oviposição na superfície da água ocorreu no quinto dia (50 ovos). 0 período de escotofase recebeu maior deposição de

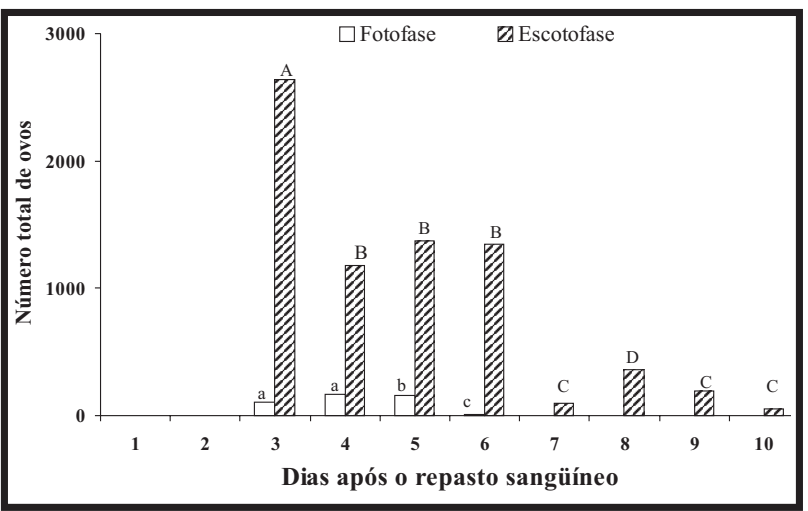

Figura 3 - Total do número de ovos depositados por Aedes aegypti em laboratório (10 repetições, Kruskal Wallis, p<0,05).

As letras minúsculas indicam diferenças significativas entre si para o período da fotofase, enquanto maiúsculas indicam diferença significativa para o período de escotofase. ovos no substrato de oviposição (5.303 ovos) do que na superfície da água (1.934 ovos).

Periodicidade de oviposição de laboratório e campo. No primeiro ciclo gonotrófico de fêmeas de $A$. aegypti, constatou-se que a oviposição em laboratório iniciou-se a partir da $1^{\text {a }}$ hora de fotofase (07:00h) (Figura 4). Os resultados demonstram que entre a $1^{\mathrm{a}}$ e $8^{\mathrm{a}}$ hora $(07: 00$ às 15:00h) o número de ovos depositados foi reduzido, não

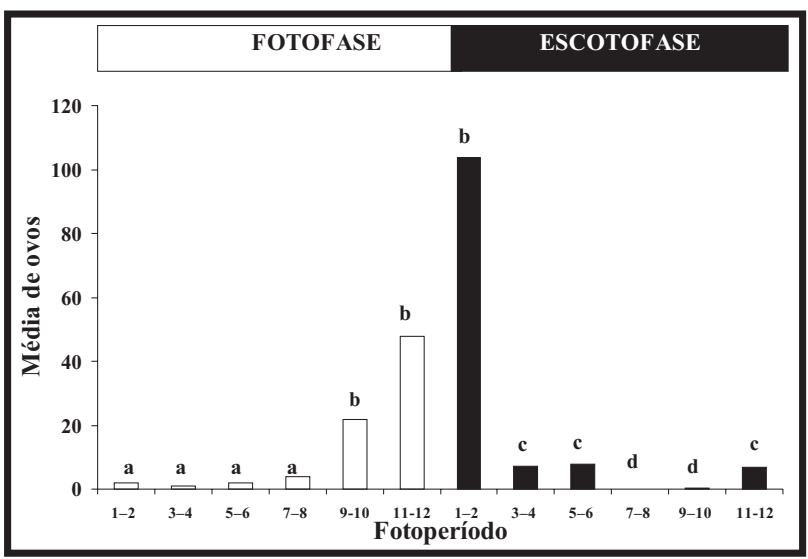

Figura 4 - Média de ovos depositados em papel de filtro por fềmeas de Aedes aegypti em laboratório, durante fotoperíodo a cada duas horas ( $N=12$ repetições para cada horário do fotoperíodo, Kruskal Wallis, $p<0,05$ ). Histogramas seguidos de letras diferentes entre si indicam diferença estatística significativa.

ocorrendo diferença significativa $(\mathrm{p}>0,05)$ entre si. No entanto, verificou-se que o horário que recebeu maior oviposição $(\mathrm{p}<0,05)$, compreendeu o período entre $9^{\mathrm{a}}$ a $12^{\mathrm{a}}$ hora $(15: 00$ 19:00h) de fotofase e a $1^{\mathrm{a}}-2^{\mathrm{a}}$ hora (19:00-21:00h) de escotofase. Subseqüientemente, a oviposição de fêmeas de A. aegypti mantevese reduzida por todo período da escotofase (Figura 4).

As coletas de ovos em campo, por meio de ovitrampas, demonstraram que na $1^{\mathrm{a}}-2^{\mathrm{a}}$ hora de fotofase ( $05: 15$ às 07:15h), não houve oviposição. A atividade de oviposição ocorreu na $3^{\mathrm{a}}-4^{\mathrm{a}}$ hora $(07: 15-09: 15 \mathrm{~h})$, reduzindo entre a $5^{\mathrm{a}}-8^{\mathrm{a}}$ hora de fotofase $(09: 15$ às 13:15h). No entanto, significativamente $(\mathrm{p}<0,05)$ maior atividade de oviposição foi observada entre a $9^{\mathrm{a}}-12^{\mathrm{a}}$ hora de fotofase (13:15 -17:15h) (Figura 5). 


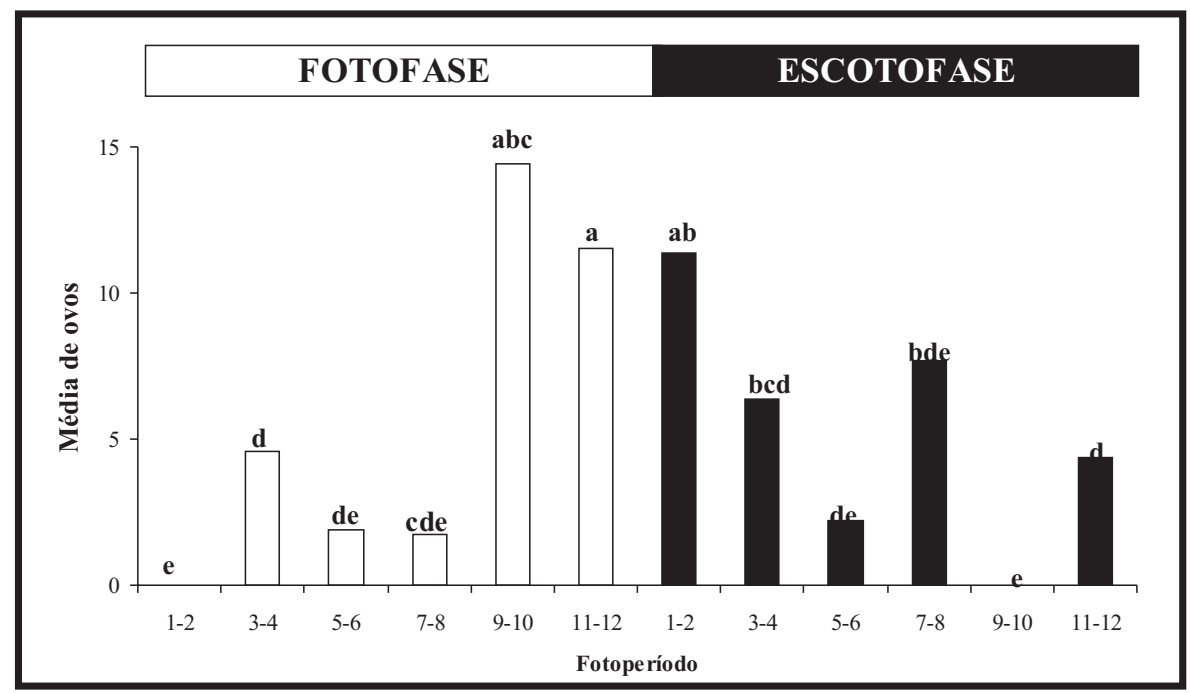

Figura 5 - Média de ovos depositados em palhetas de oviposição por fêmeas grávidas do mosquito Aedes aegypti em campo durante fotoperíodo, a cada duas horas $(N=960$ réplicas. Kruskal Wallis, $p<0,05)$.

Histogramas seguidos de letras diferentes entre si indicam diferença estatística significativa.

Na escotofase, a maior deposição de ovos foi observada na $1^{\text {a }}$ hora $(17: 15 \mathrm{~h})$ reduzindo gradativamente até a $5^{\mathrm{a}}-6^{\mathrm{a}}$ hora (21:15-23:15h). Houve uma elevada deposição de ovos na $7^{\mathrm{a}}-8^{\mathrm{a}}$ hora (23:15-01:15h). Entre a $9^{\mathrm{a}}$ e $10^{\mathrm{a}}$ hora de escotofase não foi observada deposição de ovos, a atividade de oviposição foi retomada no período da $11^{\mathrm{a}}-12^{\mathrm{a}}$ hora de escotofase (03:15-05:15h) que compreende o período crepuscular ao amanhecer (Figura 5).

\section{DISCUSSÃO}

No presente trabalho, os resultados demonstraram maior atividade de oviposição para os papéis toalha na fotofase e papel filtro na escotofase. Provavelmente, esta escolha tenha ocorrido mediante a influência de fatores físicos, tais como textura e umidade. É possível que estes papéis apresentem maior porosidade em relação aos demais substratos de oviposição, o que conseqüentemente determina uma maior concentração da umidade.

Fêmeas de Aedes aegypti apresentam preferência por depositarem seus ovos nas bordas de recipientes que tendem a armazenar água de chuva ou torneira ${ }^{1622}$. Porém, verificouse, no presente trabalho, que as fêmeas realizaram deposição de ovos diretamente na superfície da água dos recipientes de oviposição oferecidos. Estes ovos apresentaram rápida capacidade de eclosão, 30 horas no máximo, após os recipientes serem retirados das gaiolas de bioensaios. No meio ambiente, esta particularidade, provavelmente, pode assegurar ao inseto vetor rápida colonização de criadouros disponíveis, mantendo no período de estiagem uma população de insetos flutuante. Um outro aspecto importante, é que os ovos possivelmente apresentam um efeito de predação menor no ambiente em relação àqueles que aguardam as épocas das chuvas ${ }^{20}$ e menor comprometimento da sua viabilidade ${ }^{23}$. Dados como estes tornam-se relevante nos programas de monitoramento e controle de A. aegypti, quanto à remoção de criadouros remanescentes, presentes no meio ambiente.

Estudos prévios realizados por Consoli e Willians ${ }^{11}$, demonstraram que fêmeas de Aedes fluviatillis, preferiram realizar a oviposição diretamente na superfície da água, do que em superfícies de papel de filtro.

0 padrão temporal de oviposição durante o ciclo gonotrófico em A. aegypti, corroboram os resultados encontrados por Xue e Barnard ${ }^{27}$ que utilizaram Aedes albopictus, indicando o terceiro dia como o de início da oviposição no ciclo gonotrófico das fêmeas. Consoli e Willians ${ }^{11}$ indicam uma variação de 3 a 13 dias entre a alimentação sangüínea e a oviposição de A. fluviatillis, embora, maior número de ovos tenham sido depositados no dia modal. Provavelmente, estas variações são características intrínsecas de cada espécie, pois o tempo requerido entre a alimentação sangüínea e a deposição de ovos relaciona-se com fatores que dependem de controle endógeno no inseto, como a assimilação de aminoácidos essenciais que favorecem a maturação dos ovos. Estes fatores somados às condições ambientais permitem ao inseto estabelecer um ritmo circadiano, culminando com oviposiçã $0^{7}$.

A observação da periodicidade de oviposição em laboratório durante o fotoperíodo demonstrou que fêmeas de $A$. aegypti realizaram maior atividade de oviposição no período do entardecer (15:00 às 19:00h e 19:00 às 21:00h). Em laboratório, Trexler e cols ${ }^{26}$ observaram para A. albopictus um padrão diurno de oviposição, com início da atividade de deposição de ovos no período matinal (07:00h) encerandose no período noturno (22:00h). Entretanto, a atividade de oviposição do A. triseriatus foi observada durante todo 0 período do dia e da noite, mas a maior oviposição ocorreu no período crepuscular do entardecer (20:00-21:00h).

Na pesquisa de campo, a oviposição observada por fêmeas de $A$. aegypti evidencia na fotofase, o período da tarde como 0 de maior oviposição (13:15 às 17:15h) enquanto na escotofase, 
a maior atividade de oviposição foi observada no período crepuscular do entardecer (17:15 às 19:15h). Em campo, Trexler e cols ${ }^{26}$ observaram que Aedes triseriatus realizou oviposição em períodos de fotofase e escotofase, enquanto A. albopictus apenas na fotofase. Provavelmente, a variação em relação ao padrão de oviposição, mantido em laboratório e o padrão de oviposição apresentado em campo, possam estar relacionados à população de insetos disponíveis no ambiente, idade fisiológica das fêmeas, condições ambientais como temperatura e umidade relativa do ar e do período em que os experimentos foram realizados. Estes dados indicam que $A$ a agypti exibiu um padrão na periodicidade de oviposição e podem corroborar nossos resultados e inferir subsídios em programas de monitoramento e/ou controle do inseto vetor. Sugere-se, que em campo, por exemplo, armadilhas de oviposição (ovitrampa) possam ser instaladas no período de fotofase antes do anoitecer.

\section{AGRADECIMENTOS}

Ao Eduardo Ivo e Rosemary Roque nosso agradecimento pelo desenvolvimento deste trabalho.

\section{REFERÊNCIAS BIBLIOGRÁFICAS}

1. Ahmed AM, Maingon RD, Taylor PJ, Hurd H. The effects of infection with Plasmodium yoelii nigeriensis on the reproductive fitness of the mosquito Anopheles gambie. Invertebrate Reproduction and Development 36:217-222, 1999.

2. Allan SA, Kline DL. Larval rearing water and preexisting eggs influence oviposition by Aedes aegypti and Aedes albopictus (Diptera: Culicidae). Journal of Medical Entomology 35:943-947, 1998.

3. Beckel WE. Oviposition site preference of Aedes mosquitoes (Culicidae) in the laboratory. Mosquitoes News 15: 224-228, 1955.

4. Bentley MD, Day JF. Chemical ecology and behavioral aspects of mosquito oviposition. Annual Review of Entomology 34: 401-421, 1989.

5. Benzon GL, Apperson CS. Reexamination of chemically mediated oviposition behavior in Aedes aegypti (L.) (Diptera: Culicidae). Journal of Medical Entomology 25:158-164, 1988.

6. Chadee DD, Lakhan A, Ramdath WR, Persal RC. Oviposition response of Aedes aegypti mosquitoes to different concentrations of hay in Trinidad, West Indies. Journal of the American mosquito Control Association 9:346-348, 1993.

7. Clements AN. The Physiology of mosquitoes. In: Clements AN (ed) Control of Adult Activity. V 17. Pergamon Press, p. 242-260, 1963.

8. Cobert OS, Haddow AJ, Gillett JD. Observations on the oviposition-cycle of Aedes (Stegomyia) aegypti (linnaeus), IV. Annals of Tropical Medicine \& Parasitology 54:156-164,1960.

9. Consoli RAGB, Castro MMT, Silveira JN, Santos BS. Influência da coloração do substrato no comportamento de oviposição de Aedes fluviatilis (Lutz) (Diptera, Culicidae). Revista Brasileira de Entomologia 32:375-381,1988.
10. Consoli RAGB, Oliveira RL. Principais mosquitos de importância sanitária no Brasil. Fundação Oswaldo Cruz, Rio de Janeiro, 1994.

11. Consoli RAGB, Willians P. Laboratory observation on of Aedes fluviatilis (Lutz) (Diptera: Culicidae). Bulletin of Entomological Research 68: 123-136, 1978.

12. Fay RW, Perry AS. Laboratory studies of ovipositional preferences of Aedes aegypti. Mosquitoes News 24: 276-281, 1965.

13. Gillett JD, Cobert PS, Haddow AJ. Observations on the oviposition-cicle of Aedes (Stegomyia) aegypti (Linnaeus). VI. Annals of Tropical Medicine \& Parasitology 55: 427-431,1961.

14. Gillett JD, Cobert PS, Haddow AJ. Observations on the oviposition-cicle of Aedes (Stegomyia) aegypti (Linnaeus). III. Annals of Tropical Medicine \& Parasitology 53: 132-136,1958.

15. Goma LKH. Laboratory observations on the oviposition habits of Aedes (stegomyia) aegypti (Linnaeus). Annals of Tropical Medicine \& Parasitology 58:347-349, 1964

16. Gomes AC. Medidas dos níveis de infestação urbana para Aedes (Stegomyia) aegypti e Aedes (Stegomyia) albopictus em programa de vigilância entomológica. Informativo epidemiológico do Sistema Único de Saúde 7:49-57, 1998.

17. Kennedy JS. One water finding and oviposition by captive mosquitoes. Bulletin of Entomological Research 32: 279-307, 1942.

18. Kline DL. Introduction to Symposium on attractants for mosquito surveillance and control. Journal of American Mosquito Control Association 10:253-257,1994.

19. Madeira NG, Macharelli CA, Carvalho LR. Variation of the oviposition preferences of Aedes aegypti in function of substratum and humidity. Memórias do Instituto Oswaldo Cruz 97: 415-420, 2002.

20. Reiter P, Amador MA, Colon N. Enhacement of the CDC ovitrap with hay infusions for daily monitoring of Aedes aegypti populations. Journal of the American Mosquito Control Association 7: 52-55, 1991.

21. Roberts DR, Hsi BP. Method of evaluating Aedes ovipositional attractants. Journal of Medical Entomology 14:129-131, 1977.

22. Roque RA. Avaliação de armadilhas iscadas com infusões de gramíneas como atraentes e/ou estimulantes de oviposição do mosquito Aedes (Stegomyia) sp (Diptera: Culicidae). Dissertação de Mestrado, Universidade Federal de Belo Horizonte, Belo Horizonte, BH, 2002.

23. Silva HHG, Silva IG, Lira KS. Metodologia de criação, manutenção de adultos e estocagem de ovos de Aedes aegypti (Linnaeus, 1762) em laboratório. Revista de Patologia Tropical 27: 53-63, 1998.

24. Superintendência de Campanhas de Saúde Públicas (SUCAM). Resumo dos principais caracteres morfológicos do Aedes aegypti e Aedes albopictus. Ministério da Saúde, Brasília, 1989.

25. Thirapatsakun L, Tauthong P, Phanthumachinda B. Surface preferences of oviposition of Aedes aegypti in Thailand. Southeast Asian Journal of Tropical Medicine and Public Health 12: 209-212, 1981.

26. Trexler JD, Apperson CS, Schal C. Diel oviposition patterns of Aedes albopictus (Skuse) and Aedes triseriatus (Say) in the laboratory and the field. Journal of Vector Ecology 22:64-70, 1997.

27. Xue R, Barnard DR. Diel patterns of pupation, emergence, and oviposition in a laboratory population of Aedes albopictus. Journal of the American Mosquito Control Association 13: 205-207, 1997.

28. Zahiri N, Rau ME, Lewwis JD. Oviposition responses of Aedes aegypti and Aedes atropalpus (Diptera: Culicidae) females to Waters from conspecific and heterospecific normal larvae and from larvae infected with Plagiorchis elegans (Trematoda: Plangiorchiidae). Journal of Medical Entomology 34:565-568, 1997. 